\title{
Case Report: Augmentation with Blonanserin for a Schizophrenia Patient with Insufficient Response to Clozapine
}

\author{
Manabu Takaki1 ${ }^{*}$, Shigeru Takahashi², Yuji Yada1,3, Kohei Kitagawa ${ }^{3}$, Yoshiki Kishi ${ }^{3}$, \\ Norihito Yamada ${ }^{1}$ \\ ${ }^{1}$ Department of Neuropsychiatry, Okayama University Graduate School of Medicine, Dentistry and Pharmaceutical Sciences, \\ Okayama, Japan \\ ${ }^{2}$ Sekizen Hospital, Okayama, Japan \\ ${ }^{3}$ Department of Psychiatry, Okayama Psychiatric Medical Center, Okayama, Japan \\ Email: ^manabuta@cc.okayama-u.ac.jp
}

How to cite this paper: Takaki, M., Takahashi, S., Yada, Y., Kitagawa, K., Kishi, Y. and Yamada, N. (2018) Case Report: Augmentation with Blonanserin for a Schizophrenia Patient with Insufficient Response to Clozapine. Open Journal of Psychiatry, 8, 131-136.

https://doi.org/10.4236/ojpsych.2018.82012

Received: March 1, 2018

Accepted: April 20, 2018

Published: April 23, 2018

Copyright $(9) 2018$ by authors and Scientific Research Publishing Inc. This work is licensed under the Creative Commons Attribution International License (CC BY 4.0).

http://creativecommons.org/licenses/by/4.0/

\begin{abstract}
Background: Clozapine is the most efficacious among antipsychotics for patients with schizophrenia. Nevertheless, clozapine is not effective in more than about $50 \%$ of treatment refractory schizophrenia patients, and several pharmacological strategies are used to augment it. Several reviews including meta-analyses have been published, but the efficacy of augmentation therapy for clozapine-resistant patients is not adequately supported. Though there is a weak connection between the oral dose and plasma concentration of clozapine, there is no report of augmentation therapy considering the plasma concentration of clozapine. Blonanserin is reported to be effective in treatment of both positive and negative symptoms of schizophrenia and well tolerated. Methods: We obtained consent to evaluate clinical presentations and clozapine plasma concentrations at the Okayama Psychiatric Medical Center and had not identified the individual for ethical reasons. This is a case report. Results: This case fulfilled the diagnostic criteria of neuroleptic-induced dopamine supersensitivity psychosis. Monotherapy with blonanserin was not effective, but augmentation of blonanserin with clozapine was effective and well tolerated by a clozapine-resistant schizophrenia patient. Conclusion: Because clozapine may ameliorate dopamine supersensitivity psychosis, the addition of blonanserin to clozapine may be effective even if monotherapy with blonanserin was not.
\end{abstract}

\section{Keywords}

Treatment Refractory Schizophrenia, Clozapine, Blonanserin, Clozapine 


\section{Introduction}

Clozapine (CZP), one of the second-generation antipsychotics, is the most efficacious among the antipsychotics for treatment of patients with schizophrenia [1]. Nevertheless, $40 \%$ to $70 \%$ of patients defined as treatment refractory according to Kane's criteria [2] do not respond to CLZ [3] [4] [5], and several pharmacological strategies are employed to treat them [6] [7] [8]. Several reviews including meta-analyses have been published, but the efficacy of augmentation therapy for CZP-resistant patients is not well supported [6] [7] [8]. Though there is a weak relationship between the oral dose and the plasma concentration of CZP [9], there is no report of augmentation therapy based on the plasma concentration of CZP. Blonanserin (BNS) is reported to be effective in treatment of both positive and negative symptoms of schizophrenia in Japan, Korea, and China [10] [11] [12] and is well tolerated [13] [14]. Here we present a case in which supplemental BNS was effective and tolerated by a CZP-resistant schizophrenia patient and discuss it considering the pharmacological profiles of BNS, CZP concentration, and neuroleptic-induced dopamine supersensitivity psychosis (DSP).

\section{Method}

We obtained consent to evaluate clinical presentation and CZP plasma concentrations at the Okayama Psychiatric Medical Center and have not identified the individual for ethical reasons. This is a case report.

\section{Case Presentation}

A 44-year-old man started to fear he had an unpleasant body odor at 10 years old. At 14 years old, he started to experience auditory hallucinations, thought insertion, thought broadcasting, delusions of being observed, and persecution. He started high school but quit. He started several jobs, but he was able to continue only a short time because of psychiatric symptoms. At 20 years old, he consulted a hospital for the first time and was diagnosed with schizophrenia according to the Diagnostic and Statistical Manual of Mental Disorders, fourth edition (DSM-IV). He was administered a low dose of haloperidol. However, because he believed haloperidol was poison and not effective for his psychiatric symptoms, he refused it many times. We administered fluphenazine decanoate at $50 \mathrm{mg}$ per 4 weeks for 6 months, but it did not ameliorate his symptoms. We administered 20 to $40 \mathrm{mg}$ /day of olanzapine from the ages of 31 to 36 years, 24 $\mathrm{mg} /$ day of BNS from 36 to 39 years, and $8 \mathrm{mg} /$ day of risperidone from 39 to 40 years. He was able to continue a job only a short time, even at a community workshop, because of psychiatric symptoms. His psychiatric symptoms worsened, and he hit the walls and floor during hallucinations, and he continued 
walking on the road aimlessly. At 40 years old, he was hospitalized for the first time. We administered $50 \mathrm{mg}$ of risperidone long-acting injectable depot (Risperdal Consta) every two weeks with $750 \mathrm{mg} /$ day of quetiapine, and he was discharged after four months. His abnormal behavior and agitation stopped, but he was afraid of having hallucinations and hospitalized again after one month. Thus, he fulfilled the criteria for treatment refractory schizophrenia [2], and CZP was tried. His Positive and Negative Syndrome Scale (PANSS) scores were: positive 30, negative 26, total 120; Clinical Global Impressions-severity (CGI-S): 6, and Global Assessment of Functioning (GAF): 25. We increased CZP to 275 $\mathrm{mg}$ /day at one month after initiation. Because he had general myoclonus and his electroencephalogram showed general slow waves from 4 to $6 \mathrm{~Hz}$, we decreased CZP to $200 \mathrm{mg} /$ day and added $300 \mathrm{mg} /$ day of valproic acid and 50mg/day of lamotrigine. His myoclonus stopped, and he was discharged. Because he had started to smoke again, we supposed that the CZP concentration had decreased due to the CYP1A2 induction and increased CZP to $400 \mathrm{mg} /$ day. He started to work as a newspaper deliverer, but he felt stress. His PANSS scores were: positive 17, negative 15, total 62; CGI-S: 3, and GAF: 60. At 43 years old, his psychotic syndrome worsened; he drank agricultural chemicals to kill himself following hallucinations and was hospitalized. We increased CZP to $600 \mathrm{mg} / \mathrm{day}$, and his hallucinations were reduced slightly, but he became anorexic. His PANSS scores were: positive 23, negative 20, total 100; CGI-S: 5, and GAF: 30. We reduced CZP to $500 \mathrm{mg} /$ day and added $4 \mathrm{mg} /$ day of BNS. His mood improved following a reduction of hallucinations, and he was discharged. After discharge, we increased BNS to $12 \mathrm{mg}$ /day and he started working at a community workshop. His PANSS scores were: positive 16, negative 15, total 62; CGI-S: 3, and GAF: 58. His CZP concentration was $237.6 \mathrm{ng} / \mathrm{ml}$ (Okayama Psychiatric Medical Center), and his prolactin concentration was $15.90 \mathrm{ng} / \mathrm{ml}$ (normal range 4.29 - 13.69).

\section{Discussion}

Though augmenting CZP with a second antipsychotic is one of the treatments for treatment refractory schizophrenia, the efficacy of augmentation therapy for CZP-resistant patients is not well supported [6] [7] [8]. In this case, a high dose of antipsychotics was administered for a long time, and a high dose of BNS was also not effective. CZP at $200 \mathrm{mg} /$ day was effective, but he relapsed because of stress. Though CZP was increased to the maximum dose, it was not very effective and not well tolerated. Thus, this case filled the diagnostic criteria of DSP [15]. CZP may ameliorate the DSP induced by previous antipsychotic treatment because it may decrease the number of dopamine receptors [16], and augmentation with a low dose of BNS was partly effective. We may consider exclusively augmenting CZP with a second antipsychotic in the case of DSP.

In the cases of augmenting CZP with a second antipsychotic, uses of dopamine antagonists, i.e., risperidone, amisulpiride, and sulpiride are well reported but car- 
ry a risk of hyperprolactinemia, and risperidone has the risk of QTc prolongation [6] [7] [8]. BNS strongly blocks dopamine 2 (D2) receptors, 5-hydroxytryptamine (5-HT) 2 receptors, D3 receptors, and weakly blocks 5HT-2C, dopamine1 (D1), and $\alpha_{1}$ receptor activities while being almost devoid of histamine $\mathrm{H} 1$ and muscarinic M1 blocking activity [17]. The pharmacological binding profile is similar to that of sulpiride and amisulpiride except for 5-HT 2 receptor binding affinity [14]. BNS may be comparatively safer than alternative agents for sedation [13], hyperprolactinemia [14], and QTc prolongation [18]. Polypharmacy with antipsychotics is reported to increase the risk of side effects [19], and we should consider the safety profile of agents in the setting of co-administration with CZP. In this case, there was mild hyperprolactinemia but no clinical symptoms. The BNS profile may be related to its safety.

The effective concentration of CZP is reported to differ substantially between individuals [9]. Because CZP and BNS are both metabolized by CYP3A4 [17], BNS administration may increase the blood CZP concentration. However, in our case, the CZP concentration might decrease because of increasing cigarette consumption after discharge. The AGNP Consensus Guidelines for Therapeutic Drug Monitoring in Psychiatry (2011) recommend a therapeutically effective blood concentration of CZP between 350 and $600 \mathrm{ng} / \mathrm{ml}$. In our case, the blood concentration of CZP was under $350 \mathrm{ng} / \mathrm{ml}$. Because the maximum dose of CZP was not tolerable due to side effects, the CZP concentration may not have been adequate to improve his symptoms.

\section{Conclusion}

Though it is needed more cases for a clear and strong conclusion, the addition of BNS to CZP may be effective in cases of DSP, even if monotherapy with BNS is not.

\section{Conflicts of Interest}

N.Y. has received unrestricted research funding from Daiichi Sankyo, Eisai, Pfizer, Otsuka, Astellas, and Merck Sharp \& Dohme, which was deposited into research accounts at Okayama University. N.Y. has received honoraria for his participation as a speaker at educational events from UCB Japan, Tsumura, Pfizer, Dainippon-Sumitomo, Daiichi-Sankyo, Merck Sharp \& Dohme, Pfizer, Eisai, Meiji-Seika, and Mochida. M.T. has received honoraria for his participation as a speaker at educational events sponsored by Otsuka. S.K., Y.Y., K.K. and Y.K. report no additional financial or other relationship relevant to this article.

\section{Acknowledgements}

This study is partly supported by a grant from the Zikei Institute of Psychiatry.

\section{References}

[1] Leucht, S., Cipriani, A., Spineli, L., et al. (2013) Comparative Efficacy and Tolerabil- 
ity of 15 Antipsychotic Drugs in Schizophrenia: A Multiple-Treatments Meta-Analysis. Lancet, 382, 951-962. https://doi.org/10.1016/S0140-6736(13)60733-3

[2] Kane, J.M., Honigfeld, G., Singer, J., et al. (1988) Clozapine for Treatment-Resistant Schizophrenics. A Double-Blind Comparison with Chlorpromazine. Arch Gen Psychiatry, 45, 789-796. https://doi.org/10.1001/archpsyc.1988.01800330013001

[3] Chakos, M., Lieberman, J., Hoffman, E., et al. (2001) Effectiveness of Second-Generation Antipsychotics in Patients with Treatment-Resistant Schizophrenia: A Review and Metaanalysis of Randomized Trials. American Journal of Psychiatry, 158, 518-526. https://doi.org/10.1176/appi.ajp.158.4.518

[4] Lieberman, J.A., Safferman, A.Z., Pollack, S., et al. (1994) Clinical Effects of Clozapine in Chronic Schizophrenia: Response to Treatment and Predictors of Outcome. American Journal of Psychiatry, 151, 1744-1752.

https://doi.org/10.1176/ajp.151.12.1744

[5] Meltzer, H.Y., Burnett, S., Bastani, B., et al. (1990) Effects of Six Months of Clozapine Treatment on the Quality of Life of Chronic Schizophrenic Patients. Hosp. Community Psychiatry, 41, 892-897.

[6] Porcelli, S., Balzarro, B. and Serretti, A. (2012) Clozapine Resistance: Augmentation Strategies. European Neuropsychopharmacology, 22, 165-182.

https://doi.org/10.1016/j.euroneuro.2011.08.005

[7] Sommer, I.E., Begemann, M.J., Temmerman, A., et al. (2012) Pharmacological Augmentation Strategies for Schizophrenia Patients within Sufficient Response to Clozapine: A Quantitative Literature Review. Schizophrenia Bulletin, 38, 1003-1011. https://doi.org/10.1093/schbul/sbr004

[8] Taylor, D.M., Smith, L., Gee, S.H., et al. (2012) Augmentation of Clozapine with a Second Antipsychotic: A Meta-Analysis. Acta Psychiatrica Scandinavica, 125, 15-24. https://doi.org/10.1111/j.1600-0447.2011.01792.x

[9] Mauri, M.C., Volonteri, L.S., Colasanti, A., et al. (2007) Clinical Pharmacokinetics of a Typical Antipsychotics: A Critical Review of the Relationship between Plasma Concentrations and Clinical Response. Clinical Pharmacokinetics, 46, 359-388. https://doi.org/10.2165/00003088-200746050-00001

[10] Garcia, E., Robert, M., Peris, F., et al. (2009) The Efficacy and Safety of Blonanserin Compared with Haloperidol in Acute-Phase Schizophrenia: A Randomized, Double-Blind, Placebo-Controlled, Multicentre Study. CNS Drugs, 23, 615-625. https://doi.org/10.2165/00023210-200923070-00006

[11] Yang, J., Bahk, W.M., Cho, H.S., et al. (2010) Efficacy and Tolerability of Blonanserin in the Patients with Schizophrenia: A Randomized, Double-Blind, Risperidone-Compared Trial. Clinical Neuropharmacology, 33, 169-175. https://doi.org/10.1097/WNF.0b013e3181dcda50

[12] Li, H., Yao, C., Shi, J., et al. (2015) Comparative Study of the Efficacy and Safety between Blonanserin and Risperidone for the Treatment of Schizophrenia in Chinese Patients: A Double-Blind, Parallel-Group Multicenter Randomized Trial. Journal of Psychiatric Research, 69, 102-109. https://doi.org/10.1016/j.jpsychires.2015.07.015

[13] Takaki, M., Okahisa, Y., Kodama, M., et al. (2012) Efficacy and Tolerability of Blonanserin in 48 Patients with Intractable Schizophrenia. Acta Neuropsychiatrica, 24, 380-383. https://doi.org/10.1111/j.1601-5215.2012.00663.x

[14] Kishi, T., Matsuda, Y., Nakamura, H., et al. (2013) Blonanserin for Schizophrenia: Systematic Review and Meta-Analysis of Double-Blind, Randomized, Controlled Trials. Journal of Psychiatric Research, 47, 149-154. 
https://doi.org/10.1016/j.jpsychires.2012.10.011

[15] Chouinard, G. (1991) Severe Cases of Neuroleptic-Induced Supersensitivity Psychosis. Diagnostic Criteria for the Disorder and Its Treatment. Schizophrenia Reserach, 5, 21-33. https://doi.org/10.1016/0920-9964(91)90050-2

[16] Nakata, Y., Kanahara, N., Kimura, H., et al. (2017) Efficacy of Clozapine on Dopamine Supersensitivity Psychosis in Schizophrenia. International Clinical Psychopharmacology, 32, 169-173. https://doi.org/10.1097/YIC.0000000000000160

[17] Deeks, E.D. and Keating, G.M. (2010) Blonanserin: A Review of Its Use in the Management of Schizophrenia. CNS Drugs, 24, 65-84. https://doi.org/10.2165/11202620-000000000-00000

[18] Mizuki, Y. and Takaki, M. (2015) Blonanserin Ameliorated the Tendency toward QTc Prolongation Associated with Risperidone in a Patient with Schizophrenia. Journal of Clinical Psychopharmacology, 35, 101-102. https://doi.org/10.1097/JCP.0000000000000267

[19] Kishimoto, T., Watanabe, K., Uchida, H., et al. (2013) Anti-Psychotic Polypharmacy: A Japanese Survey of Prescribers' Attitudes and Rationales. Psychiatry Research, 209, 406-411. https://doi.org/10.1016/j.psychres.2013.03.014 